\title{
Processing the vane shear flow data from Couette analogy
}

\author{
Patrice Estellé ${ }^{1,{ }^{\star}}$, Christophe Lanos ${ }^{1}$, Arnaud Perrot ${ }^{2}$, Sofiane Amziane ${ }^{2}$ \\ ${ }^{1}$ LGCGM, Equipe Matériaux et Thermo-Rhéologie, Institut Universitaire Technologique, rue \\ du Clos Courtel, BP 90422, 35704 Rennes Cedex 7, France \\ ${ }^{2}$ LG2M, Université de Bretagne Sud, Rue Saint Maudé, 56321 Lorient Cedex, France \\ * Corresponding author \\ Email : patrice.estelle@univ-rennes1.fr \\ Tel : $+33(0) 223234200$ \\ Fax : +33 (0) 223234051
}

\begin{abstract}
:
A new procedure is described to convert the vane torque and rotational velocity data into shear stress vs shear rate relationships. The basis of the procedure consists in considering locally the sheared material as a Bingham fluid and computing a characteristic shear rate from Couette analogy. The approach is first applied to experimental vane data of Newtonian fluid, then used to process vane experimental data of non-Newtonian and yield stress materials. Results, which are favourably compared with torsional flow, show that the approach correctly predicts the rheological behaviour of the materials investigated.
\end{abstract}

\section{Résumé:}

Une nouvelle procédure est développée afin de convertir les données de couple et vitesse de rotation en géométrie vane sous la forme d'une relation contrainte vitesse de cisaillement. Cette procédure consiste à considérer le fluide en écoulement comme étant un fluide de Bingham, et à évaluer la vitesse de cisaillement caractéristique du fluide par une analogie de Couette. Cette approche est premièrement appliquée aux données expérimentales en géométrie vane d'un fluide newtonien, puis à celles de fluides non newtonien et à seuil d'écoulement. Les résultats, qui sont favorablement comparés à des écoulements de torsion, montrent que la procédure prédit correctement le comportement rhéologique des matériaux étudiés.

\section{Keywords:}

Vane test, rheometry, Couette analogy, Bingham model, flow curve

\section{Introduction}

Vane geometry was initially used in soil mechanics for the measurement of the shear strength of soils. It has now become a standard technique in rheometry [1], in particular to measure the yield stress of very shear-thinning liquids and structured fluids [2-7]. In this way, the vane is used in rotational controlled mode and tests are carried out at a constant and low rotational velocity. An alternative procedure is to operate the vane in a constant-stress controlled mode $[1,8]$. So, the vane is basically a creep test where wall slip is far away or subsequently reduced. Moreover, an oscillating mode may be applied to investigate 
viscoelastic properties of fluids $[9,10]$. In practice, vane tests are relatively easy to perform and the four-bladed probe may be attached on existing rheometers. With this geometry, the fluid structure is not disturbed prior the measurement, which is an advantage for sensitive fluids and semi-solid structures. Vane shear flow interpretation is commonly based on the following considerations. It is assumed that (1) the material is sheared along a cylindrical surface defined by the vane height $h$ and diameter $D$ and (2) the stress distribution is uniform over the cylindrical sheared surface. As a consequence, vane geometry appears as a Couette system, varying thickness of sheared material following material structure and cup size, where wall slip effect is eliminated. Such an analogy was previously used by Baravian et al. [10] to characterise rheological properties of gels. They found in particular an effective radius close to the height of the triangle formed by joined tips of adjacent blades. As done in this last work, Martinez-Padilla and Quemada [11] have shown that this analogy first requires the evaluation of shear factors which then allows one to calculate shear stress and shear rate.

The purpose of this work is to provide vane shear flow curves from Couette analogy, as mentioned above, for both small and wide gaps without presupposing the rheological behaviour of fluid tested. Here, the approach consists in approximating the sheared material as a Bingham fluid and computing an average shear rate in vane geometry. The applicability and the performance of the proposed approximation in vane shear flow curve determination is given and analysed from experimental data, considering Newtonian, non-Newtonian and yield stress fluids.

\section{Bingham approximation for shear rate evaluation}

Let us consider the vane geometry equivalent to Couette bob of radius $R_{b}$ and height $h . R_{c}$ is the cup radius and $\Omega$ is the rotational velocity of the vane. The fluid is assumed to be incompressible and inelastic, edge and inertia effects as well as shearing effect in the bottom zone are neglected. In Couette geometry, the shear stresses exerted on the inner and outer cylinders, respectively denoted $\tau_{b}$ and $\tau_{c}$, can be directly related to the torque, independently of the nature of the fluid, as follows:

$$
\tau_{b}(M)=\frac{M}{2 \pi h R_{b}{ }^{2}} \quad ; \tau_{c}(M)=\frac{M}{2 \pi h R_{c}^{2}}
$$


Several techniques have been developed to relate the angular velocity and the shear rate [12]. The main approximations are Power-law approximation, Krieger's methods and Newtonian approximation, which is frequently used in viscometer softwares.

Here, the stress-shear fluid behaviour is modelled by the Bingham constitutive equation, which relates the shear stress $\tau$ and the shear rate $\dot{\gamma}$ by the following relationships:

$$
\tau=K+\mu \dot{\gamma} \text { if } \tau>\mathrm{K}(2)
$$

Where $K$ is the plastic yield stress and $\mu$ is the plastic viscosity.

For a Bingham plastic model, two distinct conditions may occur in the annulus: (1) $\tau_{c} \leq K \leq \tau_{b}$; (2) $\tau_{c}>K$. The first condition implies that a rigid plug flow occurs in the region near the cup, while the second one indicates that the yield value is exceeded in the entire annulus and the fluid within the gap is fully sheared. Therefore, the rotational velocity has a separate solution corresponding to each of these two conditions.

$$
\begin{gathered}
\Omega=\frac{K}{2 \mu}\left[\ln \left(2 \pi h K R_{b}^{2}\right)-1\right]+\frac{M}{4 \pi h \mu R_{b}{ }^{2}}-\frac{K}{2 \mu} \ln M \text { if } \tau_{c} \leq K \leq \tau_{b} \\
\Omega=\frac{M}{4 \pi h \mu}\left(\frac{1}{R_{b}{ }^{2}}-\frac{1}{R_{c}{ }^{2}}\right)+\frac{K}{\mu} \ln \left(\frac{R_{b}}{R_{c}}\right) \text { if } \tau_{c}>K
\end{gathered}
$$

The derivative of Eqs. (10) and (11) with respect to the torque yields

$$
\begin{gathered}
\frac{d \Omega}{d M}=\frac{1}{4 \pi h \mu R_{b}{ }^{2}}-\frac{K}{2 \mu M} \text { if } \tau_{c} \leq K \leq \tau_{b} \\
\frac{d \Omega}{d M}=\frac{1}{4 \pi h \mu}\left(\frac{1}{{R_{b}}^{2}}-\frac{1}{{R_{c}}^{2}}\right) \text { if } \tau_{c}>K
\end{gathered}
$$

The shear rate for the Bingham plastic model can be expressed in terms of rotational velocity, torque, radius of cylinders by combining Eqs. (1), (2), (5) and (6), as follows:

$$
\begin{gathered}
\dot{\gamma}=2 M \frac{d \Omega}{d M} \text { if } \tau_{c} \leq K \leq \tau_{b} \quad \text { (7) } \\
\dot{\gamma}=2 \frac{M \frac{d \Omega}{d M}}{\left(1-\frac{R_{b}{ }^{2}}{R_{c}{ }^{2}}\right)}-\frac{\Omega .-M \cdot \frac{d \Omega}{d M}}{\ln \left(\frac{R_{b}}{R_{c}}\right)} \text { if } \tau_{c}>K
\end{gathered}
$$


Eq. (7) is the one developed by [13] in Couette geometry for a yield stress material partially sheared within the gap. One can quote that Eq. (7) does not depend on the rheological model of yield stress fluid considered. Eq. (8) is presently used to recover the shear rate for non yield stress materials or completely sheared conditions. So, as proposed in [13], the shear rate is a combination of two expressions following the flow condition in the annulus Two distinct admissible stress fields, which correspond to the shear rate solutions of eqs (7) $\&(8)$, are generated according to the flow condition within the gap. Based on the principle of virtual power [14], the appropriated stress field is the one which maximizes the energy dissipation in the flowing sample, which is obtained from the greater value of shear rate. Then, the characteristic shear rate is defined as

$$
\dot{\gamma}=\max (\text { equation(7); equation(8)) }
$$

This does not require the knowledge of the fluid yield stress value a priori and the identification of the flow regime in the annulus.

It is assumed that a series of torque measurements $M_{j}$ (corresponding to stress $\tau_{j}$ ) are made at a series of increasing (or decreasing) rotation rate $\Omega_{j}$. The derivative $\frac{d \Omega}{d M}$ is approximated by $\frac{\Omega_{j}-\Omega_{j-1}}{M_{j}-M_{j-1}}$. Once the shear rate has been estimated by Eq. (9), it is deemed to correspond to the following wall shear stress:

$$
\tau=\frac{1}{2}\left(\tau_{j}+\tau_{j-1}\right)
$$

The following remarks can be made. First, the proposed equations are constructed on the linear derivative of the angular velocity versus the applied (or measured) torque. Logarithmic derivatives are nevertheless more appropriate for logarithmic variation of the torque (or the velocity). Moreover, it should be noted that Eq. (7) is independent on the rheological law. This is not the case for Eq. (8). This equation depends on the Bingham assumption. Since this equation corresponds to a fully sheared gap, Eqs. (11) and (12) from Krieger and Elrod [15] and Krieger [16] can alternatively be used following the gap radius ratio. These later do not depend on the rheological law and might therefore be more general. As a consequence, Eq. (8) is compared here to Krieger solutions in the following to prove the relevance of the Bingham assumption in shear rate calculation to a fully sheared gap.

The first term of the Euler-Maclaurin formula approximation developed by Krieger and Elrod [15] is widely used, and takes the form of equation (11) [17]. It is noted that Krieger and 
Maron [18] have shown that this shear rate solution yields accurate results for radius ratios $\alpha$ $\leq 1.2$.

$$
\dot{\gamma}=\frac{\Omega}{\ln (\alpha)}
$$

The first term of the second Krieger solution is given by eq. (12). This shear rate solution is accurate for non-Newtonian fluids fully sheared in larger annulus, as $\alpha \leq 2$ [19].

$$
\dot{\gamma}=\left(\frac{2 \Omega}{n}\right) /\left(1-\alpha^{-2 / n}\right)
$$

With $n=d \ln \tau_{b} / d \ln \Omega$

\section{Experimental applications}

\subsection{Newtonian fluid}

Newtonian silicone oil with defined viscosity value of $98.5 \mathrm{~Pa} . \mathrm{s}$ at $25^{\circ} \mathrm{C}$ was used in these experiments. Rheological measurements were performed at $25 \pm 0.1^{\circ} \mathrm{C}$ with a Gemini 150 Malvern rheometer. Preliminary tests with plate-plate geometry of $40 \mathrm{~mm}$ in diameter and $1 \mathrm{~mm}$ in gap are performed in two replicates to check the silicone oil viscosity. Results show that the silicon oil behaves well as a Newtonian material under $25 \mathrm{~s}^{-1}$. In this shear rate range, the apparent viscosity corresponds to defined viscosity value. At higher shear rate, Newtonian behaviour is less obvious due to sample disruption at the plate-plate edge and presence of wall slip.

Vane measurement was performed with two test geometries, as detailed in Table 1. Due to the large distance between the bottom of the vanes and the base of the containers, end effects were neglected [11]. Experiments were carried out by applying linear shear ramp from 0 to $160 \mathrm{~s}^{-1}$ in $240 \mathrm{~s}$, from the rheometer software. The top of the vane was also placed at the surface of the silicon oil during experiments. The resulting torque as a function of rotational velocity shows a linear relationship, as predicted with Newtonian material when inertia forces are lower relative to the viscous forces.

For both vane geometries, shear stress as a function of shear rate of silicon oil was obtained from the procedure described above. Results, which are presented in Fig. 1, show that vane shear flow curves are in good correlation with theoretical flow curve of silicon oil in the shear rate range $10^{-3}-40 \mathrm{~s}^{-1}$, for both moderate and wide gaps. So, as shown by the results, the procedure on the two vane systems used with silicon oil correctly predicts the Newtonian 
behaviour of this fluid and its viscosity. This proves the independency of the vane morphology on the results. As the radius ratio is different with both vanes, the shear rate range obtained is also different, in particular at low shear rate. Moreover, it was checked that the shear rate of eq. (8) is always greater than the one obtained with eq. (7), as expected, due to the viscous behaviour of silicon oil.

\subsection{Non-Newtonian material}

Baravian et al. [10] have reported the flow curve of a CarboxyMethylCellulose (CMC) aqueous solution from both cone and plate and vane geometries. They have considered the effective cylinder of the vane $(9.48 \mathrm{~mm}$ in radius and $20.6 \mathrm{~mm}$ in height) calculated using various viscous materials and rotation rate, and geometric factor correction due to the nonNewtonian behaviour of the CMC. The data set of torque-rotational velocity of CMC is presently used to investigate the relevance of the proposed approach with non-Newtonian fluid. In the shear rate and shear stress calculation, we have used respectively the effective and real dimensions of the vane $(11 \mathrm{~mm}$ in radius and $17 \mathrm{~mm}$ in height). As a result, Fig.2 shows the apparent viscosity evolution of CMC against shear rate obtained from cone and plate and vane geometries. Here again, it was checked that the shear rate of eq. (8) is always greater than the one obtained with eq. (7). So, Fig. 2 shows also the comparison between the Bingham assumption and the second Krieger shear rate solution. Results, obtained from the real dimensions of the vane, show that both solutions give a similar evolution of the apparent viscosity of CMC against shear rate. However, the Bingham assumption curve is closer to the plate-plate one. This can be explained by the radius ratio which is larger than 2, as the Krieger second method is accurate for calculating shear rate from data obtained with radius ratio $\leq 2$. This illustrates the performance of the analogy with Couette geometry and the Bingham approximation in shear rate calculation. Fig.2 also shows that the effective vane dimension allows appropriated flow curve to be recovered, in particular at low shear rate. When shear rate increases, it seems that the cylindrical unsheared central zone tends to a radius close the one of the blades [20].

\subsection{Yield stress fluids}

Carbopol 940 dispersion, which is known as a yield stress fluid [21,22], was used in this experiment. A $0.15 \mathrm{wt} \%$ carbopol dispersion was prepared as follows. The dry powder which has low wetability, making it tedious to produce a homogeneous solution, is introduced in 1litre of agitated distilled water. The batch is mixed for $20 \mathrm{~min}$ until aqueous surface wetting and the dispersion of Carpobol is achieved. The obtained solution has a liquid consistancy 
and an acid ( $\mathrm{pH}$ 3.4). Then, the suspension is neutralized to $\mathrm{pH} 7$ with a common base of sodium hydroxide $(1.3 \mathrm{wt} \% \mathrm{NaOH})$. The mixing is then stopped when a visually water-clear homogenized gel suspension is achieved with air bubbles essentially absent. The $0.15 w t \%$ Carbopol suspension was placed at rest for $48 \mathrm{~h}$ at $25^{\circ} \mathrm{C}$ before rheological testing.

Rheological measurements were performed at $25^{\circ} \mathrm{C}$ using a Malvern Gemini 200 rheometer. Geometries such as a $40 \mathrm{~mm}$ plate-plate (gap $1 \mathrm{~mm}$ ), a vane attachment (vane diameter $25 \mathrm{~mm}$, vane length $37.6 \mathrm{~mm}$, cup diameter $44.3 \mathrm{~mm}$ ) as well as a Couette geometry (bob diameter $25 \mathrm{~mm}$, bob length $37.6 \mathrm{~mm}$, cup diameter $27 \mathrm{~mm}$ ) were used. Both plates, vane cup, and bob and cup cylinders are roughened to avoid slippage. Rate-controlled measurements were performed in the same shear rate range, $0-30 \mathrm{~s}^{-1}$, from the rheometer software, applying a linear up-and-down ramp. During experiments, the top of the vane and bob cylinder was placed at the fluid surface.

As a result, Figure 3 compares the apparent viscosity prediction of Carbopol between parallel plate measurement, vane and Couette data computed from the procedure described above, as well as the apparent viscosity curve obtained from the first Krieger's solution in Couette geometry. As can be seen, the three geometries compare well over the shear rate range investigated. This shows first the relevance of the proposed approximation technique in shear rate calculation with yield stress material. Moreover, we can notice the independency of system used with yields stress fluid, as vane and Couette systems provide the same result, independently of gap size and shear flow condition in the annulus. Actually, it was checked that with vane system, the fluid is always partially sheared due to the large gap used. Inversely, the Carbopol is quickly fully sheared in the Couette narrow gap. As a consequence, Bingham assumption was also here compared to the Krieger's shear rate solution. Fig. 3 shows the good correlation between these both shear rate solutions. Finally, Carpobol dispersion show yield stress and shear thinning behaviour as predicted in previous works [21,22]. It should be noted that some discrepancy between parallel plates and vane or Couette flow curves appears at low shear rate. This can be explained by the initial state of the material before testing, as no pre-shearing has been performed before the experiments.

\section{Conclusions}

In this paper we have evaluated the rheological behaviour of fluids in vane system from Couette analogy. We have used a Bingham model to locally describe the vane rotational shear flow of fluids and compute an average shear rate and the corresponding shear stress from torque-rotational velocity data. Experimental results show the correct predictions of the proposed analysis with Newtonian, non-Newtonian and yield stress materials under moderate and large gap vane systems. The developed procedure provides a practical and 
alternative method to conventional treatment of vane shear flow data. Indeed, it does not require shear factor calculations and prespecification of rheological constitutive model, as well as small or wide gap assumption. The proposed approach is also interesting because it provides a way to evaluate the material rheology of a suspension with large particles.

Acknowledgment: the authors wish to thank C. Baravian for providing the data of $\mathrm{CMC}$ solution.

\section{References}

[1] Barnes HA, Nguyen QD: Rotating vane rheometry - a review, J. Non-Newtonian Fluid Mech. 98 (2001) 1-14.

[2] Nguyen QD, Boger DV: Yield stress measurement for concentrated suspensions, J. Rheol. 27(4) (1983) 321-349.

[3] Prud'homme RK, Khan SA: Foams. Theory, measurements and applications, Marcel Dekker, Inc., New-York (1996).

[4] Rao MA, Steffe JF: Measuring yield stress of fluid foods, Food Techn. 51 (1997) 50-52.

[5] Stokes JR, Telford JH: Measuring the yield behaviour of structured fluids, J. NonNewtonian Fluid Mech. 124 (2004)137-146.

[6] Liddell PV, Boger DV: Yield stress measurement with the vane, J. Non-Newtonian Fluid Mech. 63 (1996) 235-261.

[7] Barnes HA, Carnali JO: The vane-in-cup as a novel rheometer geometry for shear thinning and thixotropic materials, J Rheol. 34(6) (1990) 841-866.

[8] Aldermann NJ, Meeten GH, Sherwood JD: Vane rheometry of bentonite gel, J. NonNewtonian Fluid Mech. 39 (1991) 291-310.

[9] Servais C, Ravji C, Sansonnens C, Bauwens I: Oscillating vane geometry for soft solid gels and foams. J Texture Studies 33 (2003) 487-504.

[10] Baravian C, Lalante A, Parker A: Vane rheometry with a large, finite gap, Appl. Rheol. 12 (2002) 81-87.

[11] Martinez-Padilla LP, Quemada D: Baffled cup and end-effects of a vane-in-a-large cup rheometer for Newtonian fluids. J. Food Eng. 80(1) (2007) 24-32.

[12] Steffe JF: Rheological methods in food engineering process, Second Edition, Freeman Press, East Lansing, USA (1996).

[13] Nguyen QD, Boger DV: Characterization of yield stress fluids with concentric cylinder viscometers, Rheol. Acta 26 (1986) 508-515.

[14] Germain P, Muller P: Introduction à la mécanique des milieux continues, Masson Edition (1979). 
[15] Krieger IM, Elrod H: Direct determination of the flow curves of non-Newtonian fluids. II. Shearing rate in the concentric cylinder viscometer, J. Appl. Phys. 24 (1953) 134-136.

[16] Krieger IM: Shear rate in the Couette viscometer, Trans. Soc. Rheol. 12 (1968) 5-11.

[17] De Hoog FR, Andersen RS: Simple and accurate formulas for flow-curve recovery from Couette rheometer data, Appl. Rheol. 16 (2006) 321-328.

[18] Krieger IM, Maron SH: Direct determination of the flow curves of non-Newtonian fluids. III. Standardized treatment of viscometric data, J. Appl. Phys. 25 (1954) 72-75

[19] Yang TMT, Krieger IM: Comparison of method for calculating shear rates in coaxial viscometers, J. Rheol. 22 (1978) 413-421.

[20] Keentok M: The measurement of yield stress of liquids, Rheol. Acta. 21 (1982) 325-332.

[21] Curran SJ, Hayes RE, Afagan A, Williams MC, Tanguy PA: Properties of carbopol solutions as model for yield-stress fluids, J. Food Sci. 67(1) (2002) 176-180.

[22] Piau JM: Carbopol gels - Elastoviscoplastic and slippery glasses made of individual swollen sponges: Meso- and macroscopic properties, constitutive equations and scaling laws J. Non-Newtonian Fluid Mech. 144(1) (2007) 1-29. 


\section{Figure captions}

Figure 1. Vane shear flow curves prediction of silicon oil compared to its theoretical flow curve.

Figure 2. Flow curves of non-Newtonian CMC solution.

Figure 3. Flow curves for $0.15 \mathrm{wt} \%$ of carbopol dispersion. 
Table captions

Table 1. Geometrical parameter of vane systems used for silicon oil experiments.

\begin{tabular}{|c|c|c|c|c|}
\hline geometry & vane radius $\mathrm{R}_{\mathrm{b}}(\mathrm{mm})$ & cup radius $\mathrm{R}_{\mathrm{c}}(\mathrm{mm})$ & Height $\mathrm{h}(\mathrm{mm})$ & Radius ratio $\mathrm{R}_{\mathrm{c}} / \mathrm{R}_{\mathrm{b}}$ \\
\hline Vane 1 & 7 & 13.5 & 30 & 1.92 \\
\hline Vane 2 & 15 & 40 & 30 & 2.67 \\
\hline
\end{tabular}




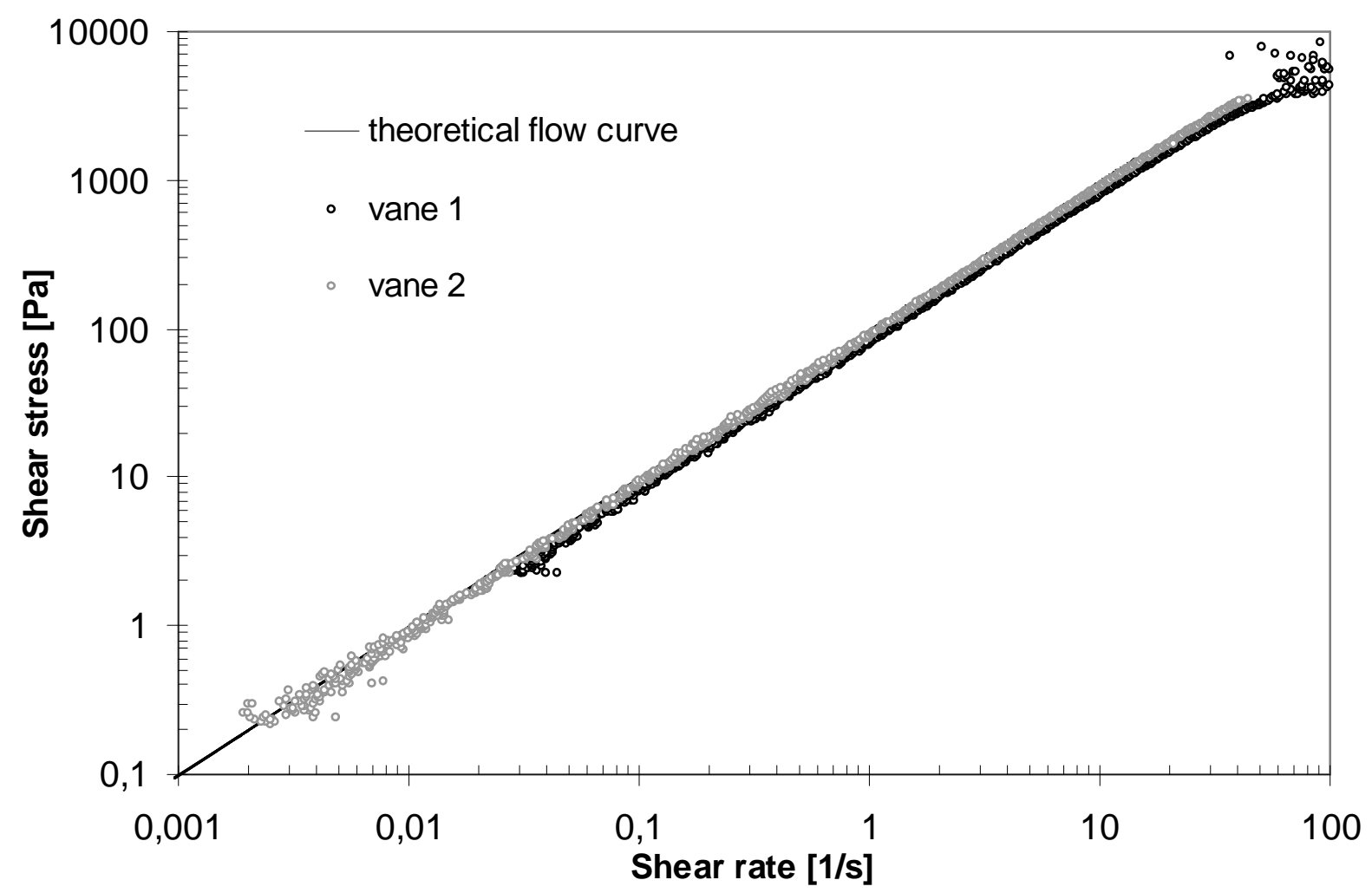

Figure 1. Vane shear flow curves prediction of silicon oil compared to its theoretical flow curve. 


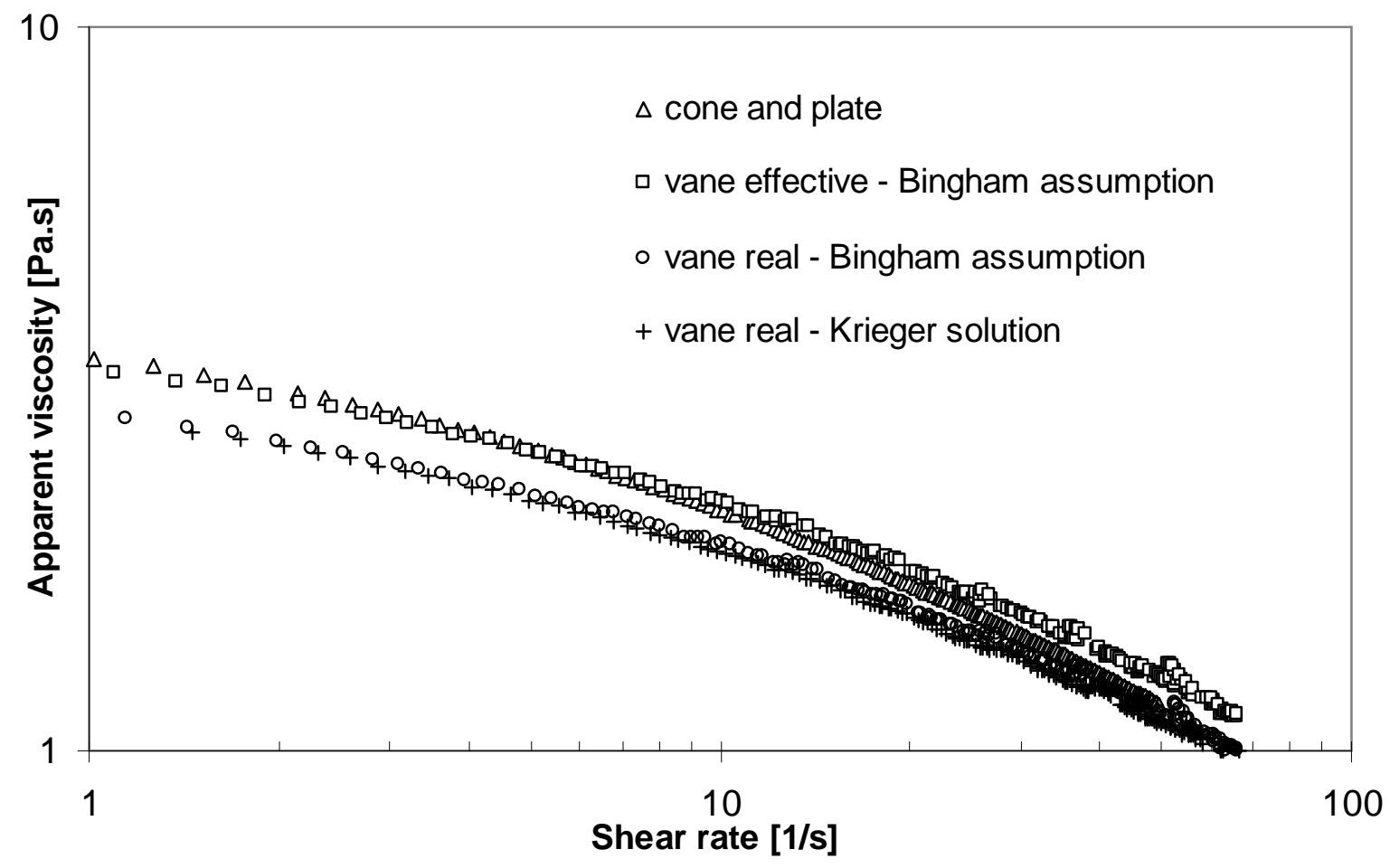

Figure 2. Flow curves of non-Newtonian CMC solution. 


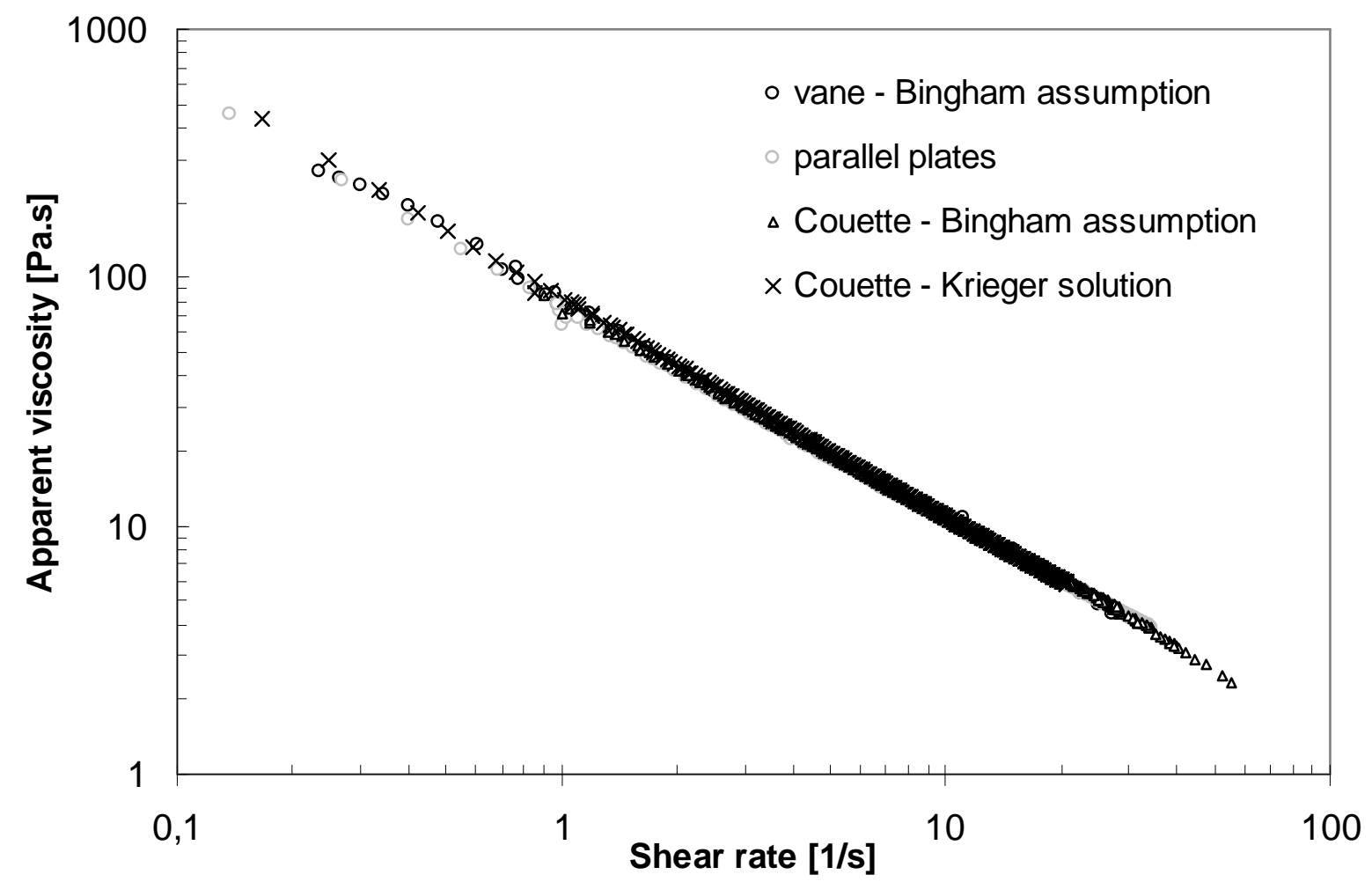

Figure 4. Flow curves for 0.15 wt $\%$ of carbopol dispersion. 